\title{
Successful Recovery of a Child with COVID-19-Induced Secondary Hemophagocytic Lymphohistiocytosis
}

\author{
Sayed Nassereddin Mostafavi ${ }^{1,2,3}$, Atefeh Sadeghizadeh ${ }^{1,}$, , Sharareh Babaei ${ }^{1}$, Rana Saleh ${ }^{1}$, Amin \\ Dehghan ${ }^{4}$, Saaman Tavakoli ${ }^{1}$ and Zahra Pourmoghaddas ${ }^{1}$ \\ ${ }^{1}$ Pediatric Department, School of Medicine, Isfahan University of Medical Sciences, Isfahan, Iran \\ ${ }^{2}$ Infectious Diseases and Tropical Medicine Research Center, Isfahan University of Medical Sciences, Isfahan, Iran \\ ${ }^{3}$ Child Growth and Development Research Center, Research Institute for Primordial Prevention of Non-Communicable Disease, Isfahan University of Medical Sciences, \\ Isfahan, Iran \\ ${ }^{4}$ Student Research Committee, School of Medicine, Isfahan University of Medical Sciences, Isfahan, Iran \\ "Corresponding author: Pediatric Department, School of Medicine, Imam Hussein Children's Hospital, Isfahan University of Medical Sciences, Imam Khomeini St, Postal Code: \\ 8195163381, Isfahan. Tel: +98-3133866266, Fax: +98-3133868286, Email: a.sadeghizade99@gmail.com
}

Received 2020 November 21; Accepted 2020 December 01

\begin{abstract}
The coronavirus disease 2019 (COVID-19) pandemic has imposed a significant burden worldwide, manifesting as a severe disease and causing mortality even in children. Severe COVID-19 disease is characterized by cytokine storm with progression to secondary hemophagocytic lymphohistiocytosis (sHLH). We describe an 18-month-old boy in Iran, previously healthy, diagnosed with COVID19-induced sHLH. Three weeks after close contact with COVID-19 confirmed cases, he was admitted with high fever, lethargy, mild respiratory distress, skin rash, and conjunctivitis with swollen eyelids and lips. Laboratory data revealed elevated levels of erythrocyte sedimentation rate (ESR), C-reactive protein (CRP) and liver enzymes, and mild thrombocytopenia. His clinical condition rapidly deteriorated, with septic shock, hepatosplenomegaly, and respiratory failure. Laboratory tests showed cytopenia, coagulopathy, hyperferritinemia, and hypertriglyceridemia, which met the criteria for sHLH diagnosis. Chest computed tomography (CT) revealed bilateral infiltrations that suggested acute respiratory distress syndrome (ARDS) of COVID-19 that was confirmed by a positive realtime polymerase chain reaction (RT-PCR) test. Therefore, the child was treated with intravenous immunoglobulin (IVIG), glucocorticoid, hydroxychloroquine, lopinavir/ritonavir, and interferon $\beta$-1a. This therapeutic strategy enabled complete recovery from fever, regaining consciousness, weaning from respiratory support, and resolving shock. Serial chest radiographs showed diminishing infiltrations. Sequential physical examinations revealed an overall significant reduction in spleen and liver span. Laboratory data showed rapid improvement from cytopenia and coagulopathy, normalization of liver enzyme levels, and reduction in hyperinflammation markers. Although ARDS is the most common cause of death from COVID-19, other complications such as sHLH may be lethal; thus, early diagnosis and appropriate treatment are necessary for saving patients' lives.
\end{abstract}

Keywords: COVID-19, Hemophagocytic Lymphohistiocytosis, Children

\section{Introduction}

Coronavirus disease 2019 (COVID-19) has spread throughout the world and was declared a pandemic by the World Health Organization (WHO) (1). Although the proportion of children infected by COVID-19 seems comparatively lower, and the rate of severe symptomatic illness is relatively less than that of adults, the disease in some children could become critical and severe $(2,3)$.

Severe COVID-19 is characterized by a viral phase and then the cytokine storm, which causes acute respiratory distress syndrome (ARDS), multiorgan failure, and death. Cytokine storm is an uncontrolled systemic inflammatory response resulting from the release of large amounts of pro-inflammatory cytokines and chemokines, which induces an under-recognized condition, namely secondary hemophagocytic lymphohistiocytosis (sHLH). Affected patients present with rapid onset of fever, cytopenia, coagulopathy, elevated transaminase levels, hyperferritinemia, and multiorgan dysfunction (4). Treatment consists mainly of glucocorticoids, intravenous immunoglobulin (IVIG), and chemotherapy $(5,6)$. Despite these interventions, the mortality rate is as high as $20 \%$. Anticytokine strategies and type I interferons have been proposed to combat the hyperinflammation state, potentially reducing mortality from this condition (7).

In two adults with COVID-19-induced SHLH, the outcomes were poor (8). Recent publications have revealed 
a manifestation of COVID-19 in children that is similar to Kawasaki disease shock syndrome, but no previous cases in children with COVID-19 have been reported in association with sHLH (3). However, an 18-month-old boy in Isfahan, Iran, has survived COVID-19-induced sHLH. We report the clinical course and therapeutic interventions in this case.

\section{Case Presentation}

Written informed consent to treatment was obtained from the patient's parents.

On March 6, 2020, an 18-month-old Iranian child, from Isfahan, without any underlying disease, was admitted to a hospital for high fever, poor feeding, diarrhea, and drowsiness. He had previously been completely healthy; he had become ill seven days after he received routine national vaccinations, which included DPT, MMR, and OPV. The parents reported close contact with COVID-19-confirmed cases in his grandparents three weeks earlier.

He was lethargic and irritable at the time of admission. Physical examination revealed mild bilateral nonpurulent conjunctivitis, mild respiratory distress, and hypoxemia. Laboratory findings revealed absolute lymphopenia, mild thrombocytopenia, increased C-reactive protein (CRP) level, increased erythrocyte sedimentation rate (ESR), and significant elevations in the levels of aspartate transaminase (AST) and alanine transaminase (ALT) (Table 1). Because the child had received high doses of acetaminophen during the previous 3 days, acetaminophen toxicity was initially suspected; however, the serum level of acetaminophen was in the normal range. Kawasaki disease was suspected, but echocardiography revealed a normal left ventricular ejection fraction and no evidence of coronary artery involvement. To confirm COVID-19, chest computed tomography (CT) was performed, and samples from the nasopharynx were subject to real-time polymerase chain reaction (RT-PCR) (9). Findings on chest CT were not suggestive of COVID-19 pneumonitis (Figure 1A). On the second day, the patient's condition deteriorated. Severe edema developed in the eyelids and lips without peripheral edema; a faint macular erythematous rash appeared over the forehead and back (Figure 2), septic shock ensued, respiratory distress and hypoxemia worsened, and hepatosplenomegaly was confirmed by sonography. Shock was managed according to the guidelines of the American College of Critical Care Medicine (10), with aggressive fluid therapy, epinephrine drip, and empirical antibiotic therapy with meropenem and vancomycin in the pediatric intensive care unit. In addition, a single dose of methylprednisolone $(20 \mathrm{mg} / \mathrm{kg}$ ) was injected to prevent possible severe allergic reactions.

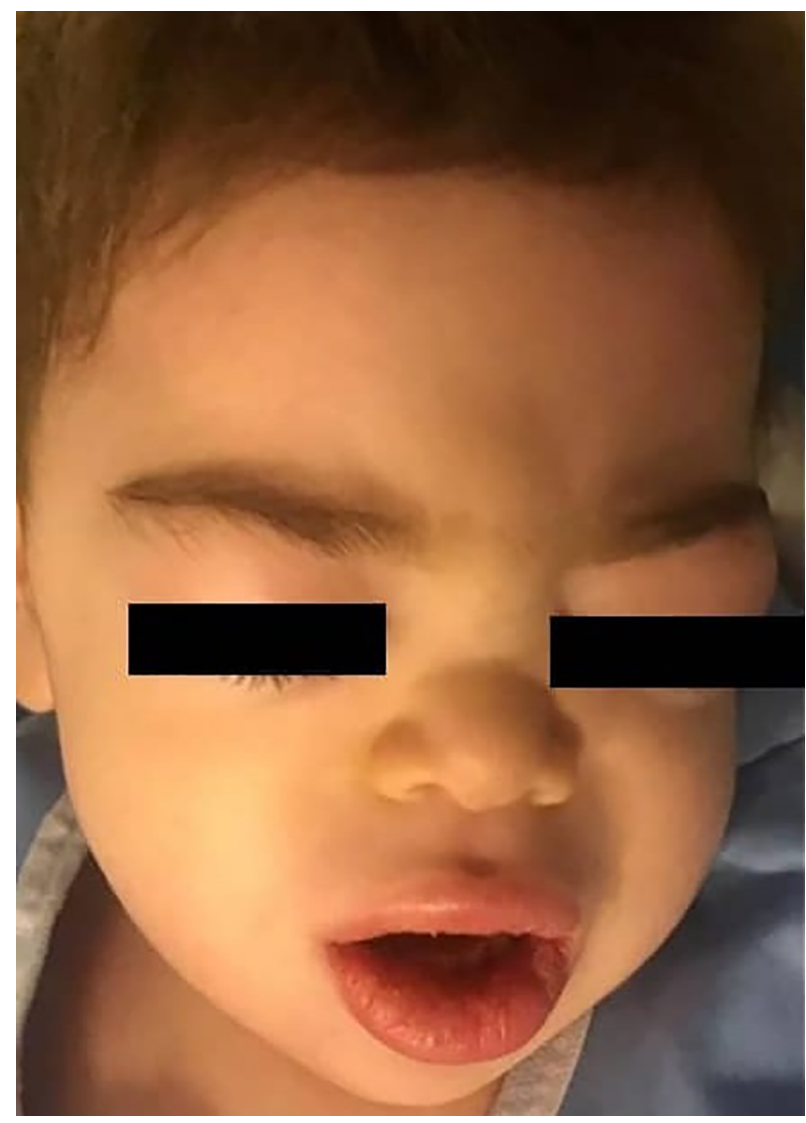

Figure 1. Facial edema: Swollen lips and eyelids of an 18-month-old boy with coronavirus disease 19 (COVID-19)-induced secondary hemophagocytic lymphohistiocytosis (sHLH) at the time of admission.

On the third day, the patient's general condition rapidly deteriorated further, with severe respiratory distress and hypoxemia, a decrease in consciousness level, severe irritability, and upper gastrointestinal bleeding. Additional laboratory tests revealed a progressive decrease in platelet counts and hemoglobin level, hypofibrinogenemia, hypertriglyceridemia, and hyperferritinemia (Table 1), which suspected the presence of SHLH. Bone marrow aspiration and biopsy were not done due to the unstable condition of the child and non-adherence of the parents. NK cell activity and soluble CD25 were not evaluated because of insufficient laboratory facilities. The level of interleukin6(IL-6) was in the normal range $(4.09 \mathrm{pg} / \mathrm{mL}$; normal range: $\leq 7 \mathrm{pg} / \mathrm{mL}$ ). New chest CT showed peripheral ground-glass opacities and also complete consolidation in the upper and middle thirds of the thorax, which was highly suggestive of ARDS of COVID-19 (Figure 1B). The results of PCR indicated severe acute respiratory syndrome coronavirus 2 (SARS-CoV-2) infection. 


\begin{tabular}{|c|c|c|c|c|c|c|c|c|c|}
\hline & Day1 & Day2 & Day3 & Day4 & Day6 & Days & Day10 & Day14 & Ten Days After Discharge \\
\hline pSOFA score & 7 & . & 15 & & & 7 & & 2 & \\
\hline $\begin{array}{l}\text { Organ systems that } \\
\text { failed }\end{array}$ & $\begin{array}{l}\text { Respiratory, coagulation, } \\
\text { renal, neurologic }\end{array}$ & - & $\begin{array}{l}\text { Respiratory, coagulation, } \\
\text { renal, hepatic } \\
\text { cardiovascular neurologic }\end{array}$ & & & $\begin{array}{l}\text { Respiratory, coagulation, } \\
\text { renal, hepatic neurologic }\end{array}$ & & Respiratory renal & \\
\hline $\begin{array}{l}\text { WBC }(\text { reference } 3700 \text { - } \\
10000 \times 10^{3} \text { cells } / \mu \mathrm{L} \text { ) }\end{array}$ & 6000 & 16600 & 12700 & 15600 & 13300 & 8200 & 6600 & 10500 & 13300 \\
\hline Neutrophil,\% & 73.8 & 77 & 77.1 & 65.1 & 70.2 & 65 & 69 & 70 & 79.3 \\
\hline Lymphocyte,\% & 20.8 & 16.9 & 20.9 & 29.4 & 25.6 & 27 & 27 & 25 & 15.1 \\
\hline $\begin{array}{l}\text { Hemoglobin (reference } \\
10.5-13.5 \mathrm{~g} / \mathrm{dL} \text { ) }\end{array}$ & 11.2 & 9.1 & 6.9 & 7.9 & 11.2 & 10.6 & 10.6 & 10 & 10.3 \\
\hline $\begin{array}{l}\text { Platelet count (reference } \\
150.0-450.0 \times 10^{3} \\
\text { cells } / \mu \mathrm{L} \text { ) }\end{array}$ & 103000 & 46000 & 23000 & 86000 & 103000 & 119000 & 141000 & 269000 & 306000 \\
\hline $\begin{array}{l}\text { AST (reference 15.0 - } 42.0 \\
\text { IU/mL) }\end{array}$ & 499 & 195 & 107 & 53 & 52 & 40 & - & - & 29 \\
\hline $\begin{array}{l}\text { ALT (reference 15.0 - } 42.0 \\
\text { IU/mL) }\end{array}$ & 391 & 182 & 129 & 105 & 87 & 63 & - & - & 62 \\
\hline T-bilirubin, $\mathbf{m g} / \mathbf{d L}^{\mathbf{a}}$ & 0.4 & . & 1.7 & . & . & 1.7 & . & 0.8 & \\
\hline INR & 1.34 & - & 1.2 & 2 & $>5$ & 2.7 & 1.9 & 1 & 1 \\
\hline $\begin{array}{l}\text { D-dimer(reference up to } \\
500 \mathrm{ng} / \mathrm{mL} \text { ) }\end{array}$ & - & - & $>8000$ & . & - & $>6000$ & - & 4100 & 300 \\
\hline $\begin{array}{l}\text { Fibrinogen (reference } \\
200-400 \mathrm{mg} / \mathrm{dL} \text { ) }\end{array}$ & - & - & 101 & - & . & 155 & - & 140 & 147 \\
\hline $\begin{array}{l}\text { FDP(reference }<\mathbf{5} \\
\mu \mathrm{g} / \mathrm{mL})\end{array}$ & - & - & $>130$ & - & - & 25 & - & - & \\
\hline $\begin{array}{l}\text { Triglycerides (normal } \\
\text { fasting }<265 \mathrm{mg} / \mathrm{dL} \text { ) }\end{array}$ & . & . & 271 & 277 & - & - & 123 & - & \\
\hline $\begin{array}{l}\text { Ferritin (reference 7- } 140 \\
\text { ng/mL) }\end{array}$ & - & - & 703 & - & . & - & 1650 & 1035 & 199 \\
\hline $\begin{array}{l}\text { Lactate dehydrogenase } \\
\text { (reference up to } 850 \mathrm{IU} / \mathrm{L} \text { ) }\end{array}$ & - & - & 1036 & - & 1479 & - & 950 & - & 459 \\
\hline $\begin{array}{l}\text { C-reactive protein } \\
\text { (reference up to } 6 \mathrm{mg} / \mathrm{L} \text { ) }\end{array}$ & 54 & - & - & . & 21 & - & 18 & 11 & \\
\hline $\begin{array}{l}\text { ESR 1st h }(\text { reference }< \\
15 / \text { st } h)\end{array}$ & 36 & . & - & - & & - & 3 & - & 10 \\
\hline Procalcitonin, $\mu \mathrm{g} / \mathbf{L}$ & 10 & . & . & - & & - & . & . & \\
\hline Creatinin, $\mathbf{m g} / \mathbf{d L}^{\mathbf{a}}$ & 0.7 & 0.6 & 0.5 & 0.6 & 0.6 & 0.5 & 0.5 & 0.5 & \\
\hline
\end{tabular}

Treatment with intravenous immunoglobulin (IVIG) $(0.5 \mathrm{~g} / \mathrm{kg} /$ day in four doses $)$ and dexamethasone $\left(10 \mathrm{mg} / \mathrm{m}^{2}\right.$ for two weeks) was initiated. Also, COVID-19 therapy, including hydroxychloroquine and lopinavir/ritonavir, was prescribed. Respiratory support with biphasic positive airway pressure was administered via nasal mask to maintain oxyhemoglobin saturation between $88 \%$ and $92 \%$ without endotracheal intubation. On the fourth day, because his condition remained serious, a single dose of interferon $\beta$ 1a was injected subcutaneously.

During the following seven days, the clinical and laboratory test results of the patient gradually improved. Fever abated on the fifth day of hospitalization. Irritability decreased, and the level of consciousness continuously improved from the sixth day. Respiratory support was decreased from the fifth day of hospitalization, and epinephrine drip was discontinued on the seventh day. Weaning of non-invasive ventilation (NIV) was initiated from the 7th day of admission, and the child remained in oxygen therapy with mask on the eleventh day and in ambient air from the thirteenth day. Repeated chest radiographs showed a significant decrease in infiltrations in both lung fields (Figure 3). Hepatosplenomegaly improved significantly from the eleventh day on. At discharge on the fourteenth day, the liver was palpable $1 \mathrm{~cm}$ below the costal margin, and the spleen was nonpalpable. Laboratory tests showed progressive improvement in platelet count, C-reactive protein level, prothrombin time, international normalized ratio, aspartate transaminase level, and alanine transaminase level, but the ferritin and lactate dehydrogenase levels remained high until discharge. Antiviral and antibiotic therapy was discontinued on the tenth day of hospitalization, and the child was discharged in good condition 14 days after admission with prescription of dexamethasone in tapering doses.

He remained isolated at home for 14 days under close 

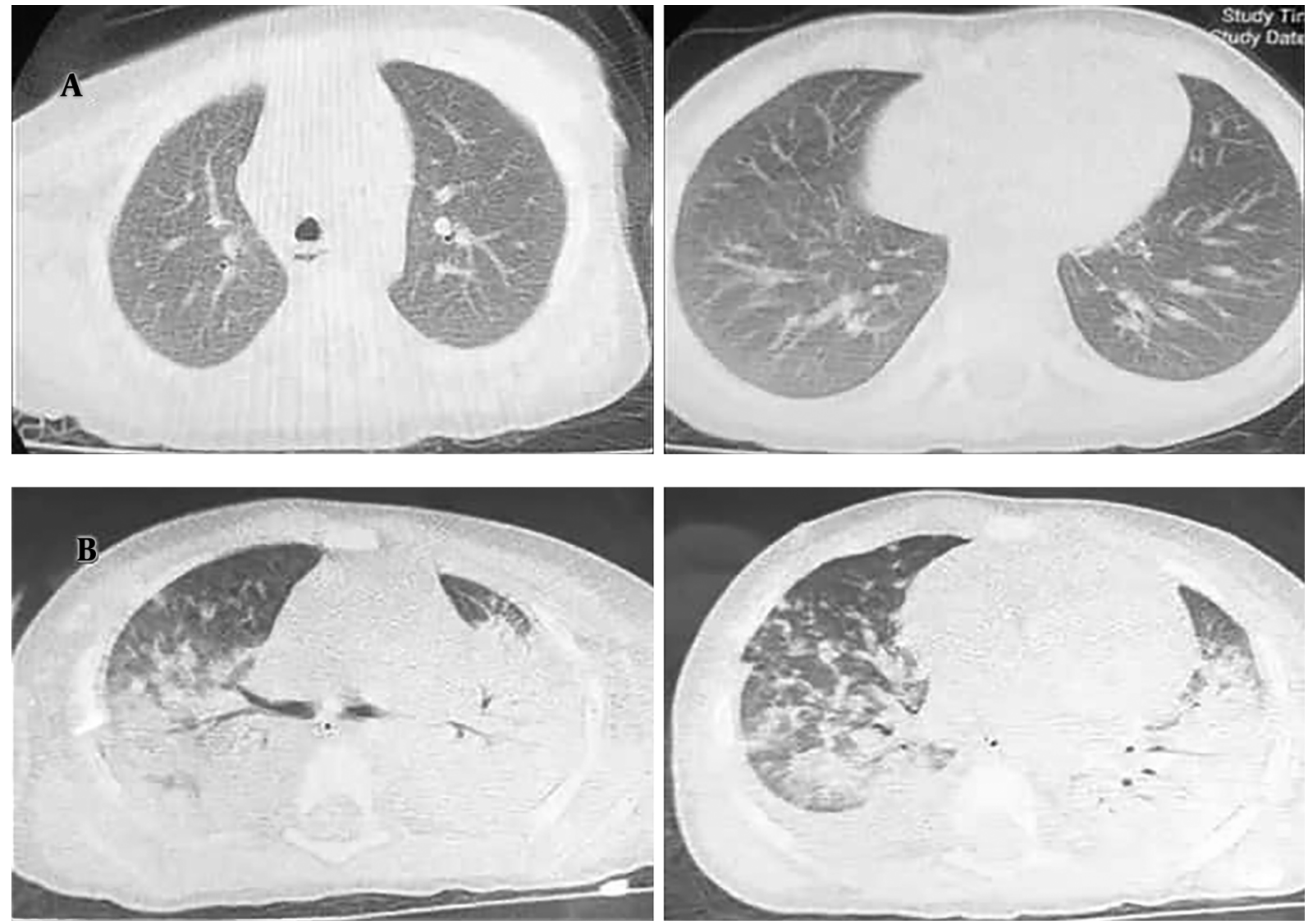

Figure 2. Chest computed tomographic manifestations: Axial slices in an 18-month-old boy with coronavirus disease 19 (COVID-19)-induced secondary hemophagocytic lymphohistiocytosis (sHLH) on the first and third days of hospitalization. A, Scan on the first day of hospitalization, demonstrating no lesions in the lungs; B, scan on the third day of hospitalization, demonstrating bilateral infiltration suggestive of acute respiratory distress syndrome of COVID-19.

medical observation. Ten days after discharge, he was in good condition without additional symptoms. Laboratory tests revealed a decrease in ferritin and D-dimer without cytopenia, and his recovery was considered successful. Table 1 summarizes the trends in laboratory findings and adapted pediatric Sequential Organ Failure Assessment scores (11).

\section{Discussion}

We presented the first child with COVID-19-induced HLH and completely recovered with appropriate treatments as a result of early diagnosis. He presented with a high fever, drowsiness, conjunctivitis, and mild respiratory distress, and hypoxemia. His condition progressively deteriorated, met five out of eight criteria of sHLH (5).

To save the life of this patient, in addition to treating COVID-19 according to the national protocol (12), the treatment was based on standard sHLH protocols. The core of HLH treatment is immunosuppressive therapy and chemotherapy. In addition to dexamethasone or etoposide, IVIG is an appropriate supplement in patients with viral infection $(5,6)$. COVID-19 has imposed a significant medical burden worldwide, and Iran is one of the countries early involved in the pandemic with severe cases (13), even in children $(14,15)$.

The viral particles of COVID-19, especially in respiratory mucosa, stimulate activation and proliferation of CD8 and natural killer $\mathrm{T}$ lymphocytes. The massive release of cytokines by CD8 promotes the multiplication of histiocytes and their differentiation into macrophages, which cross the endothelium to pass into the tissues. Macrophages, in turn, release pro-inflammatory cytokines (IL-1, IL-6, and tumor necrosis factor- $\alpha$ [TNF- $\alpha]$ ) that maintain the activation of CD8 T lymphocytes. In this way, a cascade of immune responses is triggered, which leads to cytokine release syndrome and progress to sHLH (16). A recent report in the UK has been noted an unusual presentation 

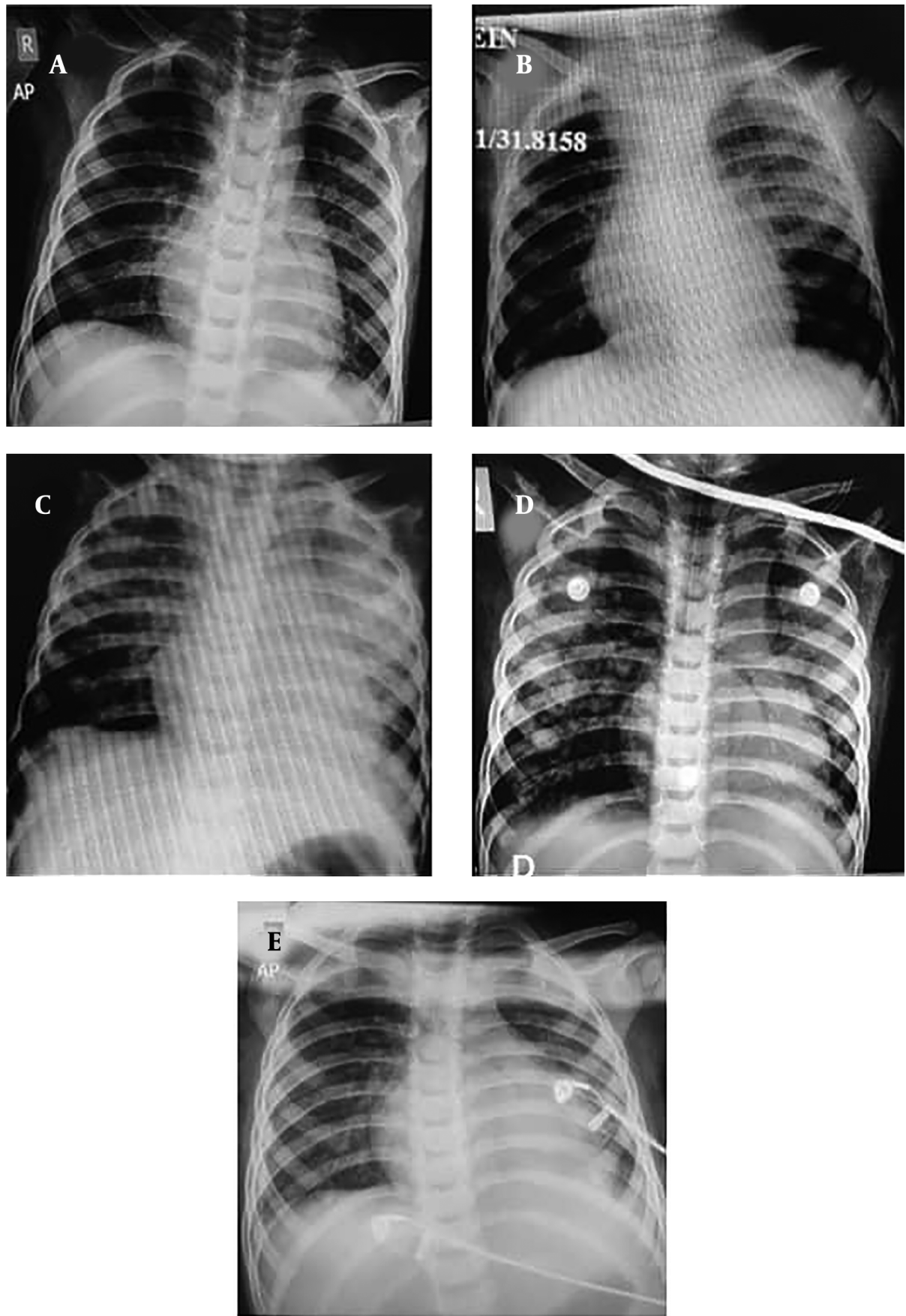

Figure 3. Chest radiographic manifestations: Serial chest radiographs of an 18-month-old boy with coronavirus disease 19 (COVID-19)-induced secondary hemophagocytic lymphohistiocytosis (sHLH). Images on the A, first; B, third; C, fourth; D, sixth; and E, tenth days of hospitalization, showing rapid diminishing of infiltration.

in several children similar to atypical Kawasaki disease or Kawasaki disease shock syndrome, but none of them met sHLH criteria (3).

Immunosuppressive agents such as steroids, IVG, and selective cytokine blockade have been suggested to treat these conditions; however, their safety and efficacy have not been verified. COVID-19-induced SHLH has been reported only in two adults with elevated IL-6 levels, which 
was treated with tocilizumab. Both developed multiorgan failure and died. The patient had received neither the treatment of sHLH nor interferon (8). Because our patient had a normal level of IL-6, tocilizumab was not prescribed.

Type I interferon plays a significant role in antiviral immunity by interfering with viral replication and slowing cell metabolism or secretion of cytokines. Interferon- $\beta$ is a more potent inhibitor of coronaviruses (17). In China's national protocol for the treatment of COVID-19 in children, interferon- $\alpha$ nebulization is recommended in severe cases (18). According to the Iranian COVID-19 guideline, interferon $\beta$-1a has been suggested for critical COVID-19 cases (13). We decided to administer a single dose of interferon $\beta$-1a as an adjuvant therapy with sHLH and antiviral treatment after his clinical condition deteriorated.

Although several forms of COVID-19 treatment are being tested in randomized trials, no drug treatment is currently established. The combination of hydroxychloroquine, lopinavir/ritonavir, and interferon $\beta$-1a is being studied in a clinical trial (code: ID IRCT20100228003449N28) (19).

Adverse effects of the MMR vaccine include fever, lymphadenopathy, joint pain, and hypersensitivity reactions; however, sHLH and multiorgan failure have not been previously reported (20). In the present patient, the immune response to the MMR vaccine might lead to an abnormal immune response to the SARS-CoV-2.

The use of interferon $\beta$-1a in addition to high-dose dexamethasone and IVIG and antiviral agents coincided with the reversal of our patient's critical condition. After three days of the treatment, the fever abated, consciousness improved, irritability lessened, the need for respiratory support decreased, and the shock abated. In addition, a decrease in chest radiographic infiltration, reductions in liver and spleen sizes, and improvement in laboratory test results demonstrated that cytokine release and macrophage activation had been well controlled.

The COVID-19 pandemic has caused the deaths of many people, mostly from respiratory manifestations. However, other manifestations and complications such as HLH are life-threatening, that early diagnosis and appropriate treatments can save the patients' lives.

\section{Footnotes}

Authors' Contribution: Study concept: NM, AS, and ZP. Data gathering: SB, RS, AD, and ST. Drafting the manuscript: $\mathrm{AD}$ and $\mathrm{RS}$. Critical revision of the manuscript for important intellectual contents: NM, AS, and ZP, SB, and ST.

Conflict of Interests: All authors have no conflict of interest to declare.
Funding/Support: This research received no specific grant from funding agencies in public, commercial, or non-profit sectors.

Informed Consent: An informed consent form was obtained from the parents of the patient.

\section{References}

1. World Health Organization. Multisystem inflammatory syndrome in children and adolescents with COVID-19. 2020.

2. Dong Y, Mo X, Hu Y, Qi X, Jiang F, Jiang Z, et al. Epidemiology of COVID-19 Among Children in China. Pediatrics. 2020;145(6). doi: 10.1542/peds.2020-0702. [PubMed: 32179660].

3. Riphagen S, Gomez X, Gonzalez-Martinez C, Wilkinson N, Theocharis P. Hyperinflammatory shock in children during COVID-19 pandemic. Lancet. 2020;395(10237):1607-8. doi: 10.1016/S0140-6736(20)31094-1. [PubMed: 32386565]. [PubMed Central: PMC7204765].

4. Mehta P, McAuley DF, Brown M, Sanchez E, Tattersall RS, Manson JJ, et al. COVID-19: consider cytokine storm syndromes and immunosuppression. Lancet. 2020;395(10229):1033-4. doi: 10.1016/S0140-6736(20)30628-0. [PubMed: 32192578]. [PubMed Central: PMC7270045].

5. Henter JI, Horne A, Arico M, Egeler RM, Filipovich AH, Imashuku S, et al.HLH-2004: Diagnostic and therapeutic guidelines for hemophagocytic lymphohistiocytosis. Pediatr Blood Cancer. 2007;48(2):124-31. doi: 10.1002/pbc.21039. [PubMed: 16937360].

6. Halyabar O, Chang MH, Schoettler ML, Schwartz MA, Baris EH, Benson LA, et al. Calm in the midst of cytokine storm: a collaborative approach to the diagnosis and treatment of hemophagocytic lymphohistiocytosis and macrophage activation syndrome. Pediatr Rheumatol Online J. 2019;17(1):7. doi: 10.1186/s12969-019-0309-6. [PubMed: 30764840]. [PubMed Central: PMC6376762].

7. Huang C, Wang Y, Li X, Ren L, Zhao J, Hu Y, et al. Clinical features of patients infected with 2019 novel coronavirus in Wuhan, China. Lancet. 2020;395(10223):497-506. doi: 10.1016/S0140-6736(20)30183-5. [PubMed: 31986264]. [PubMed Central: PMC7159299].

8. Radbel J, Narayanan N, Bhatt PJ. Use of Tocilizumab for COVID-19Induced Cytokine Release Syndrome: A Cautionary Case Report. Chest. 2020;158(1):e15-9. doi: 10.1016/j.chest.2020.04.024. [PubMed: 32343968]. [PubMed Central: PMC7195070].

9. Corman VM, Landt O, Kaiser M, Molenkamp R, Meijer A, Chu DK, et al. Detection of 2019 novel coronavirus (2019-nCoV) by real-time RT-PCR. Euro Surveill. 2020;25(3). doi:10.2807/1560-7917.ES.2020.25.3.2000045. [PubMed: 31992387]. [PubMed Central: PMC6988269].

10. Davis AL, Carcillo JA, Aneja RK, Deymann AJ, Lin JC, Nguyen TC, et al. American College of Critical Care Medicine Clinical Practice Parameters for Hemodynamic Support of Pediatric and Neonatal Septic Shock. Crit Care Med. 2017;45(6):1061-93. doi: 10.1097/CCM.0000000000002425. [PubMed: 28509730].

11. Matics TJ, Sanchez-Pinto LN. Adaptation and Validation of a Pediatric Sequential Organ Failure Assessment Score and Evaluation of the Sepsis-3 Definitions in Critically Ill Children.JAMA Pediatr. 2017;171(10). e172352. doi: 10.1001/jamapediatrics.2017.2352. [PubMed: 28783810]. [PubMed Central: PMC6583375].

12. Karimi A, Rafiei Tabatabaei S, Rajabnejad M, Pourmoghaddas Z, Rahimi H, Armin S, et al. An Algorithmic Approach to Diagnosis and Treatment of Coronavirus Disease 2019 (COVID-19) in Children: Iranian Expert's Consensus Statement. Arch Pediatric Infect Dis. 2020;8(2) doi: 10.5812/pedinfect.102400.

13. Jamaati H, Dastan F, Tabarsi P, Marjani M, Saffaei A, Hashemian SM A Fourteen-day Experience with Coronavirus Disease 2019 (COVID19) Induced Acute Respiratory Distress Syndrome (ARDS): An Iranian Treatment Protocol. Iran J Pharm Res. 2020;19(1):31-6. 
14. Rahimzadeh G, Ekrami Noghabi M, Kadkhodaei Elyaderani F, Navaeifar MR, Enayati AA, Manafi Anari A, et al. COVID-19 Infection in Iranian Children: A Case Series of 9 Patients. J Pediatrics Rev. 2020:139-44. doi 10.32598/jpr.8.2.139.

15. Eghbali A, Shokrollahi S, Mahdavi NS, Mahdavi SA, Dabbagh A. COVID 19 in pediatric patients: A case series. J Cell Mol Anesth. 2020;5(1):3-5.

16. Li X, Geng M, Peng Y, Meng L, Lu S. Molecular immune pathogenesis and diagnosis of COVID-19. J Pharm Anal. 2020;10(2):102-8. doi: 10.1016/j.jpha.2020.03.001. [PubMed: 32282863]. [PubMed Central: PMC7104082].

17. Deng X, Yu X, Pei J. Regulation of interferon production as a potential strategy for COVID-19 treatment. arXiv preprint arXiv:2003.00751.2020.
18. Shen KL, Yang YH. Diagnosis and treatment of 2019 novel coronavirus infection in children: a pressing issue. World J Pediatr. 2020;16(3):21921. doi: 10.1007/s12519-020-00344-6. [PubMed: 32026147]. [PubMed Central: PMC7091265].

19. Lythgoe MP, Middleton P. Ongoing Clinical Trials for the Management of the COVID-19 Pandemic. Trends Pharmacol Sci. 2020;41(6):363-82. doi: 10.1016/j.tips.2020.03.006. [PubMed: 32291112]. [PubMed Central: PMC7144665].

20. Kuter BJ, Brown M, Wiedmann RT, Hartzel J, Musey L. Safety and Immunogenicity of M-M-RII (Combination Measles-MumpsRubella Vaccine) in Clinical Trials of Healthy Children Conducted Between 1988 and 2009. Pediatr Infect Dis J. 2016;35(9):1011-20. doi: 10.1097/INF.0000000000001241. [PubMed: 27254037]. 